\title{
Persistência Intergeracional de Educação no Brasil: O Caso da Região Metropolitana do Recife
}

\author{
Michela Barreto Camboim Gonçalves \\ Pesquisadora - Fundação Joaquim Nabuco - Campus Anísio Teixeira \\ Endereço para contato: Rua Dois Irmãos, 92 - Apipucos - Recife - PE \\ CEP: 52071-440 - E-mail: michela.camboim@fundaj.gov.br \\ Raul da Mota Silveira Neto \\ Professor - Universidade Federal de Pernambuco - UFPE \\ Endereço para contato: Avenida dos Economistas, s/n - Cidade Universitária - Recife - PE \\ CEP: 50740-590 - E-mail: rau.silveira@uol.com.br
}

Recebido em 22 de abril de 2012. Aceito em 06 de maio de 2013.

\begin{abstract}
Resumo
A partir de uma base em dados primária derivada de pesquisa de campo da Fundação Joaquim Nabuco, este trabalho preenche parte da lacuna na literatura brasileira ao apresentar evidências recentes e inéditas a respeito da mobilidade intergeracional de educação na Região Metropolitana do Recife em 2010, evidências estas que são cotejadas com aquelas para demais Regiões Metropolitanas obtidas para o ano de 1996. Os principais resultados obtidos, que refletem um período de significativas transformações sociais, confirmam o que a literatura que trata da mobilidade intergeracional de educação destaca como importante: a persistência de educação entre pais e filhos no Brasil ainda é muito alta e heterogênea, mesmo em 2010 no caso do Recife, embora apresente redução em relação ao ano de 1996. A probabilidade de um filho cujo pai não tem escolaridade replicar o status educacional do seu pai é de $30 \%$, valor considerado elevado quando tantas oportunidades educacionais estão disponíveis.
\end{abstract}

\section{Palavras-Chave}

educação, persistência intergeracional, armadilha de pobreza

\begin{abstract}
The main objective of this work is to verify if the social changes in Brazil in recent decades impacted positively in increased rates of intergenerational mobility of education. Since based on the PNAD data of 1996's, Brazil is cited as one of the countries with the lowest intergenerational mobility in income and education in the world. Thus, this study provides results on intergenerational mobility of education in the metropolitan areas of Brazil in 1996, with results to a more recent period for the Metropolitan Region of Recife in 2010, based on data from Fundaj. The main results presented here confirm what the persistence of education among parents and children in Brazil is very high and heterogeneous. The probability of a child whose father has no education to replicate the educational status of his father is $30 \%$, very high value for the year when so many educational opportunities are available.
\end{abstract}




\section{Keywords}

education, intergeneration persistence, mobility, poverty trap

\section{JEL Classification}

R13, R28, Z13

\section{Introdução}

Ao menos ao longo de todo o período histórico para o qual se dispõe de informações confiáveis, um dos problemas econômicos marcantes do Brasil tem sido sua má distribuição de renda. Com um coeficiente de Gini para a distribuição da renda total familiar per capita, cuja média durante as últimas duas décadas oscilou em torno de $0,59,{ }^{1}$ o Brasil continua ocupando posição de destaque internacional como uma das sociedades mais desiguais do planeta, a despeito da melhora registrada na primeira década deste século. Durante o mesmo período, a média latino-americana ficou entre 0,49 e 0,50, e a africana entre 0,43 e 0,47. Em regiões mais igualitárias, como o grupo dos países ricos (a OCDE), o mesmo índice não ultrapassou a média de $0,34{ }^{2}$

De acordo com Soares (2006), a partir de 2001 a desigualdade de renda no Brasil passa a apresentar uma tendência inédita de queda, de modo que, em 2004, a desigualdade é a menor já medida pela Pesquisa Nacional por Amostra de Domicílios (PNAD) em toda sua história. ${ }^{3}$ Apesar disso, Barros et al. (2006) mostram que a proporção da renda apropriada pelos 10\% mais pobres era maior no final da década de 1970 e início da década de 1980 do que atualmente. Desta forma, conforme o relatório publicado em 2006 pelo Programa das Nações Unidas para o Desenvolvimento [PNUD, (2006)], a desigualdade no Brasil medida pelo índice de Gini é menor apenas que na latino-americana Guatemala e que nos países africanos Suazilândia, República Centro-Africana, Serra Leoa, Botsuana, Lesoto e Namíbia. Ou seja, em 2006, o Brasil era o oitavo país mais desigual do mundo.

Numa situação em que a desigualdade entre ricos e pobres e entre grupos sociais é ampla, uma preocupação fundamental dos pesquisadores diz respeito à existência de oportunidades para que uma

${ }^{1}$ Como se sabe, situado entre zero e um, o índice de Gini reflete níveis maiores (menores) quando se aproxima de um (zero).

2 Veja-se Deininger e Squire (1996).

3 Resultado também consistente com Barros et al. (2006). 
pessoa que se encontre num estado inicial relativamente desfavorecido se desloque para uma posição melhor. Neste contexto, se for verdade que, em geral, filhos de pais ricos tendem a ser ricos e, por outro lado, filhos de pais pobres tendem a ser pobres, então, até que ponto se pode dizer que a desigualdade é transmitida entre gerações? Ademais, será que se pode afirmar que o grau de persistência de status econômico entre gerações é diretamente relacionado com o grau de persistência da desigualdade ao longo do tempo?

De acordo com Lam (1999), a baixa mobilidade intergeracional de renda e de educação são duas das causas do alto grau de desigualdade que existe no Brasil. Para explicar a consequência para a sociedade da combinação entre desigualdade e falta de mobilidade, Friedman (1962) argumenta que ao se comparar duas sociedades com o mesmo grau de desigualdade, aquela com o sistema mais rígido, ou em que a família permanece sempre na mesma posição social apresenta uma desigualdade menos desejável do que aquela sociedade que apresenta maior mobilidade, já que um maior grau de mobilidade é considerado pela sociedade um sinal de possibilidade de igualdade de oportunidades.

Parte da literatura que estuda as fontes intergeracionais da desigualdade brasileira tem como foco o papel do "background familiar" na determinação de salários (Lam e Schoeni, 1993), na mobilidade intergeracional de educação (Ferreira e Veloso, 2003b; Barros et al. 2001; Black e Devereux, 2010) e de ocupação (Pastore e Silva, 1999). De uma maneira geral, a conclusão desses trabalhos é que, principalmente no Brasil, há significativa transmissão de status social entre as gerações, o que contribui para a persistência das desigualdades. Por exemplo, Behrman et al. (1999) destacaram que a mobilidade intergeracional educacional no Brasil é a menor da América Latina e uma das menores do mundo. Já em Ferreira e Veloso (2003b) a mobilidade educacional na região Nordeste do Brasil é significativamente menor que a da região Sudeste. Segundo os autores a grande diferença entre as duas regiões está na persistência de educação no grupo de filhos de pais sem escolaridade, ou seja, no Nordeste a probabilidade de o filho de um pai sem escolaridade permanecer sem escolaridade é de 53,9\%, no Sudeste esse número é equivalente a $21,2 \%$.

4 Leia-se "características familiares". 
Por sua vez, Dunn (2004) encontrou estimativas que colocaram o Brasil no topo da lista dos países com maior imobilidade intergeracional de renda.

Pode-se argumentar, entretanto, que estes resultados não são retratos atuais da sociedade brasileira, uma vez que todas estas estimativas utilizam os dados da PNAD de 1996, a última fonte de dados onde é possível obter informações sobre mobilidade intergeracional. De 1990 até hoje, o Brasil passou por intensas transformações socioeconômicas, destacando-se a abertura econômica, a estabilização da moeda, a criação, intensificação e massificação de programas sociais como Bolsa Família, a valorização do salário mínimo e a expansão para oferta de serviços de educação, entre outros. Todos estes fatores, isolados ou em conjunto, proporcionaram mudanças significativas na estrutura social do país, com resultados já visualizados por alguns indicadores, como a queda recente da desigualdade identificada por Soares (2006) e Hoffmann (2006), por exemplo. Por esta razão, no cenário internacional, há expectativas de que o Brasil de hoje apresente níveis de mobilidade intergeracional de status social muito mais significantes do que aqueles obtidos pelos acima referidos autores que anteriormente estudaram o assunto com base nos dados da PNAD de 1996.

Neste sentido, visando superar parte desta lacuna nos estudos de mobilidade intergeracional, o presente estudo tem como objetivo fornecer estimativas recentes a respeito dos níveis de mobilidade educacional brasileira, considerando-se especificamente a situação da Região Metropolitana do Recife (RMR) em 2010. Tal tarefa se fez exequível em decorrência da disponibilidade de utilização de um banco inédito de microdados primários produzidos pela Fundação Joaquim Nabuco - Fundaj. De forma complementar e para permitir um cotejo com os resultados atuais para a RMR, a pesquisa apresenta também resultados sobre a mobilidade educacional nas Regiões Metropolitanas (RMs) do Brasil em 1996 que, a despeito de serem as localidades mais dinâmicas do país, ${ }^{5}$ não mereceram a atenção dos pesquisadores da área.

Para consecução desse objetivo, o trabalho foi estruturado da seguinte forma. $\mathrm{Na}$ seção dois apresenta-se uma revisão teórica sobre mobilidade intergeracional. Discute-se o conceito de mobilidade in-

5 Em termos de oportunidades de emprego, renda, quantidade e qualidade das escolas. 
tergeracional e as principais medidas encontradas na literatura na seção três do trabalho. Já na seção quatro, encontram-se evidências empíricas disponíveis sobre a mobilidade educacional no Brasil e no mundo. Os dados utilizados no trabalho são apresentados e discutidos na seção cinco. Na seção seis, são apresentadas e discutidas as estimativas obtidas na pesquisa. Por fim, na seção sete, encontram-se as considerações finais.

\section{Mobilidade Intergeracional na Teoria Econômica}

Seguindo a tradição econômica, os estudos de mobilidade social usualmente comparam as diferenças de renda entre pais e seus filhos (quando ficam adultos). Os primeiros estudos enfatizaram o papel do altruísmo das famílias em investir no capital humano das gerações futuras. Ou seja, o aspecto fundamental é que as famílias conscientemente agem para garantir que a posição de seus filhos seja maximizada no futuro. Tais estratégias incluem a tomada de decisões em relação à instrução e educação que são mediadas pelas dotações naturais (como talento e aptidões) e pelas oportunidades disponíveis na sociedade e na sorte. ${ }^{6}$

Diversos estudos apresentam modelos explicando a relação entre desigualdade de renda e nível educacional associado ao processo de acumulação de capital humano. ${ }^{7}$ Em Bichernall (2001), o ritmo de acumulação de capital humano está associado a choques técnicos e ao nível educacional dos pais que, por sua vez, está relacionado diretamente com a forma como a renda é distribuída. Em linhas gerais, o modelo desenvolvido pelo autor sugere a existência de um equilíbrio intergeracional na distribuição de renda e de capital humano que seria decorrente de uma inter-relação entre o estoque de capital humano dos pais e dos filhos e de como este é influenciado pelo ambiente global, sensível a choques tecnológicos, e pelo ambiente local, que se refere ao efeito dos pais nas decisões de investimento de capital humano dos filhos.

\footnotetext{
6 Veja-se Becker e Tomes (1979, 1986).

7 Veja-se Galor e Zeira (1993).
} 
A ideia básica do modelo de Bichernall (2001) é a de que existe uma persistência das características socioeconômicas dos pais sobre a trajetória educacional de seus filhos e esta é afetada pela localização espacial da família. Segundo o autor, é de se esperar que em regiões com baixo dinamismo econômico o efeito familiar dos pais de baixa qualificação prevaleça em relação ao ambiente externo. O modelo teórico também sugere que os ambientes mais suscetíveis a avanços tecnológicos tendem a apresentar uma maior mobilidade de renda em relação a regiões com menor dinamismo econômico.

No modelo de Checchi (2006), as escolhas educacionais de uma geração afetam as escolhas das gerações futuras. A principal contribuição deste modelo em relação a outros é que ele apresenta um enfoque muito mais amplo sobre a questão da mobilidade intergeracional ao introduzir explicitamente a importância de fatores financeiros, culturais e sociais sobre a persistência intergeracional. A hipótese subjacente ao modelo é que indivíduos com níveis educacionais mais elevados melhoram seus prospectos futuros em termos de probabilidade de emprego, salário esperado e qualidade do trabalho, assim como aumentam as chances de que seus filhos também tenham níveis educacionais mais elevados.

O modelo parte do pressuposto de que as escolhas educacionais das pessoas são condicionadas por suas habilidades individuais que são não observáveis (como o talento), pelo background cultural e recursos financeiros da família, pelos recursos públicos e pelo capital social. $\mathrm{O}$ aspecto chave desse pressuposto é de que a maioria desses fatores apresenta persistência intertemporal e intergeracional. $\mathrm{O}$ autor considera que as características genéticas são importantes para a posição social de uma pessoa. O caráter hereditário de tais características as torna especiais, e podem colocar alguns indivíduos em posição de vantagem em detrimento a outros. Tais características englobam a habilidade (não observável), raça, altura, cor dos olhos, inteligência, e assim por diante. Apesar da dificuldade de separar os efeitos que são genéticos dos traços culturalmente adquiridos, ${ }^{8}$ as evidências empíricas obtidas de amostras de gêmeos indicam que a contribuição relativa da genética à persistência intergeracional é

8 Uma das formas utilizadas na literatura para captar especificamente o efeito das características genéticas sobre o desempenho educacional, desempenho no mercado de trabalho e sobre os salários e etc., é a partir da utilização de amostra de gêmeos (Ashenfelter e Krueger, 1992; Pronzato, 2012; Holmlund et al. 2011). Plug (2004) sugere uma alternativa para estimar o efeito "puro" do background cultural das famílias a partir de uma amostra de filhos adotados. 
baixa. ${ }^{9} \mathrm{~A}$ ideia da influência cultural ou social sobre a transmissão intergeracional de status socioeconômico deriva do fato de que filhos de pais mais escolarizados têm também mais chances de terem níveis educacionais mais elevados, devido, por exemplo, ao acesso à informação sobre a qualidade das escolas, de modo que esses pais podem orientar seus filhos a escolherem àquelas que fornecerem as melhores oportunidades. ${ }^{10}$ Checchi (2006) argumenta ainda que pais escolarizados são mais conscientes do valor psicológico e econômico da educação e, por isso, pressionam mais seus filhos a alcançarem níveis educacionais mais elevados. O elemento motriz que justifica a persistência intergeracional de background cultural entre as famílias é a escolha matrimonial. Becker (1993) é um dos primeiros estudos a enfatizar a homogeneidade do nível educacional dos casais, relacionando-a com persistência intergeracional de status socioeconômico.

O terceiro canal propulsor da persistência intergeracional é a restrição de crédito. $\mathrm{O}$ argumento de Checchi é que, se a educação é limitada pelos recursos financeiros familiares, e por outro lado, níveis educacionais mais elevados habilitam os indivíduos ao acesso a empregos com melhor remuneração, então, esses dois fatores atuando em conjunto proporcionam as condições necessárias que criam e reforçam a armadilha de pobreza: famílias pobres são privadas de investir na educação dos filhos por falta de recursos e inabilidade de acesso aos mercados financeiros, de modo que seus filhos permanecem não escolarizados (ou pouco escolarizados) e pobres e também inabilitados a investir na educação dos seus filhos. ${ }^{11}$

Do ponto de vista empírico, não é fácil distinguir entre influências culturais e financeiras, uma vez que a educação e a renda são correlacionadas. Entretanto, a medida da persistência intergeracional do quantil mais rico da população (que não apresenta restrição de liquidez) fornece uma indicação aproximada da extensão de persistência intergeracional atribuída ao background cultural [Grawe, (2004); Couch e Lillard, (2004)].

9 Bowles e Gintis (2002) mostram que os resultados dos testes de QI apresentam pouca relação com os níveis de salário, e usam esta evidência para concluir que a contribuição da habilidade para a persistência intergeracional deve ser baixa.

${ }^{10}$ Shavit e Blossfeld (1993) apresentam resultados de treze países sobre a persistência intergeracional da desigualdade de oportunidades no acesso educacional.

${ }^{11}$ Grawe (2004) e Couch e Lillard (2004) sugerem através de evidências empíricas que a persistência intergeracional de renda é maior nos extremos da distribuição, o que de fato reforça a ideia de armadilha de pobreza e armadilha de riqueza. 
O quarto e último fator propulsor da persistência intergeracional definido por Checchi refere-se à segregação territorial, estando correlacionada com a riqueza familiar. Para o autor, se as escolhas residenciais são influenciadas pela avaliação da qualidade da escola local e infraestruturas do bairro, e tais infraestruturas afetam o preço dos imóveis, então famílias ricas ganharão acesso às melhores escolas por estarem próximas a elas. Melhor qualidade da escola combinada com uma vizinhança culturalmente mais homogênea proporcionará um maior capital social, ${ }^{12}$ que claramente representará uma maior vantagem para as crianças criadas em tal ambiente.

As conclusões obtidas pelo modelo de Checchi (2006) são consistentes com as evidências empíricas encontradas para os países em desenvolvimento [Grawe, (2004)]: a mobilidade intergeracional será menor se os retornos educacionais são altos ou a pobreza é generalizada (e, portanto, a restrição de liquidez é alta). Similarmente, a mobilidade de nível educacional deveria aumentar caso as escolas fossem capazes de reduzir o impacto do background familiar sobre o sucesso educacional.

\section{Mobilidade Intergeracional: Definições e Estratégias de Mensu- ração}

Existem vários aspectos a serem considerados para definição do conceito de mobilidade social. Em Aldridge (2003), de um modo geral, mobilidade social pode ser definida como o movimento ou oportunidade para movimento entre diferentes classes sociais ou diferentes grupos ocupacionais. Para Heath e Payne (1999), uma sociedade aberta ou flexível é aquela em que seus indivíduos podem se mover livremente, como resultado de fatores como aptidão, inteligência, habilidade e esforço, para uma escala social superior à que ocupava na infância. Behrman (2000), por outro lado, define que mobilidade social é o movimento em determinado período de tempo, seja de indivíduos, grupos ou famílias, em respeito a algum indicador socioeconômico.

12 A definição sintética de capital social de Bourdieu (1980) consiste na soma de recursos atuais ou potenciais, adquiridos através de uma rede de relações de familiaridade ou institucionalizada e que podem promover ganhos tanto econômicos, quanto sociais aos agentes que interagem nesta rede. 
É convencional distinguir entre mobilidade ascendente e descendente (que é o movimento para cima ou para baixo na hierarquia de privilégios) e intergeracional de intrageracional e mobilidade na carreira. ${ }^{13}$ Formalmente, mobilidade intrageracional é o movimento de indivíduos entre diferentes classes sociais ao longo da sua vida; mobilidade intergeracional é a posição social alcançada por um adulto comparada com a posição de seus pais; mobilidade absoluta é a mudança na estrutura da sociedade, ou seja, é a mudança na distribuição da população entre as diferentes classes sociais; e mobilidade relativa é o movimento de um indivíduo entre diferentes classes sociais, independentemente de mudanças na distribuição da população.

Checchi e Dardanoni (2002) fazem a distinção entre os movimentos relativos e absolutos inseridos no contex to de mobilidade intergeracional. Os autores argumentam que a mobilidade absoluta entre gerações está relacionada à distância entre as distribuições marginais da variável socioeconômica de pais e filhos, em termos de localização e dispersão. Já movimentos relativos entre as gerações estão relacionados à existência de uma relação positiva entre as posições que pais e filhos ocupam em suas respectivas distribuições marginais, se houve troca relativa de posições ou não. Mais especificamente, o interesse deste trabalho é voltado à observação de movimentos relativos de educação entre pais e filhos.

A observação de movimentos relativos é relevante, mesmo que se trate da simples mudança de posição de indivíduos em períodos diferentes. A razão é que esses movimentos tendem a estar associados à sensação de uma sociedade mais justa, no sentido de que indivíduos mais habilidosos terão suas habilidades reconhecidas e irão alcançar posições melhores que seus pais na distribuição. Entretanto, esse reconhecimento e a troca de posições na distribuição podem depender de características herdadas e recursos transferidos dos pais para os filhos, que influenciam a posição que os últimos podem alcançar na distribuição total.

É necessário acrescentar que existe uma diferenciação no conceito de mobilidade social empregado pela Sociologia e pela Economia. De acordo com o conceito empregado pela Sociologia, encontrado nos estudos de Pastore (1979 e 1986), Pastore e Zylberstajn (1996),

${ }^{13}$ Referência: A Dictionary of Sociology. John Scott and Gordon Marshall. Oxford University Press, 2005. 
Pastore e Silva (1999) e Scalon (1999), uma sociedade apresenta maior mobilidade que outra se os filhos na primeira sociedade têm uma probabilidade maior de pertencer a uma classe social diferente da dos seus pais. Por outro lado, o conceito usado por muitos economistas, e neste estudo, identifica que uma sociedade apresenta maior mobilidade que outra se a classe social dos pais na primeira sociedade tem uma importância menor na determinação da classe social dos filhos.

Por conta de tal diferenciação, é possível encontrar resultados de que a mobilidade é alta no primeiro conceito e baixa na segunda definição. De fato, Pastore (1979), utilizando-se da PNAD de 1973, e Pastore e Silva (1999), utilizando-se da PNAD de 1996, encontraram que a mobilidade social no Brasil é elevada, com cerca de mais de $60 \%$ dos filhos em posição social diferente da posição social dos pais. Todavia, os autores também observam que a educação e o status ocupacional do pai são fatores muito importantes na determinação do status ocupacional do filho, o que caracteriza baixa mobilidade na definição empregada por este trabalho.

A abordagem padrão para a análise da mobilidade intergeracional baseia-se na mensuração do desempenho dos pais em algum indicador socioeconômico e procura inferir a importância deste desempenho sobre a performance do filho, capturada a partir do mesmo indicador. No caso deste estudo, estamos interessados em saber a importância da educação do pai na determinação da educação do filho, o que comumente é chamado de persistência intergeracional de educação. Outros sinônimos serão utilizados neste estudo: persistência educacional, mobilidade intergeracional de educação ou apenas mobilidade educacional. A transferência de sucesso entre gerações determina o que é chamado de persistência intergeracional, já que uma parcela herdada dos pais é que compõe a característica socioeconômica do filho. A partir dessa relação entre características de pais e filhos, uma variedade de medidas foram propostas para tentar avaliar qual a magnitude da influência dos pais para a determinação das características dos filhos e suas consequências para a igualdade de oportunidades na sociedade. As principais medidas de mobilidade intergeracional encontradas nos estudos empíricos serão utilizas neste trabalho: regressões de persistência por Mínimos Quadrados Ordinários (MQO) e por Regressões Quantílicas. Tais metodologias serão detalhadas a seguir. 


\subsection{Regressões de Persistência}

A maior parte dos trabalhos que estuda a mobilidade educacional o faz a partir da estimação da reação da escolaridade do filho em relação à escolaridade dos pais, ou seja, a partir de estimação de uma medida de elasticidade. Este trabalho segue inicialmente este padrão. Formalmente, isto corresponde à estimação dos parâmetros de uma regressão com a seguinte especificação:

$$
\begin{aligned}
y_{f i}= & \alpha+\beta y_{p i}+\varepsilon_{i} \\
\text { onde: } & E\left(\varepsilon_{i}\right)=0 \\
& E\left(\varepsilon_{i} y_{p i}\right)=0 \\
& E\left(\varepsilon_{i}^{2}\right)=\sigma_{\varepsilon}^{2}
\end{aligned}
$$

onde $\boldsymbol{y}_{f i}$ representa o logaritmo da educação do filho da família $\boldsymbol{i}, \boldsymbol{y}_{p i}$ representa logaritmo da educação do pai da família $\boldsymbol{i}$ e $\boldsymbol{\varepsilon}_{\boldsymbol{i}}$ é um termo estocástico. $\mathrm{O}$ coeficiente $\beta$ mede o grau de persistência intergeracional de educação. A medida 1- $\beta$ é chamada de grau de mobilidade intergeracional de educação. Quando $\beta=1$, a expectativa da família em relação à educação dos filhos é mantida entre as gerações, ou seja, a persistência intergeracional é máxima e a mobilidade é inexistente. No outro extremo, quando $\beta=0$, a educação dos filhos é idêntica para todos os níveis de educação dos pais, sendo a persistência intergeracional inexistente e a mobilidade máxima. Em outras palavras, a interpretação correta deste coeficiente aponta que, quanto mais perto da unidade, maior a influência do "background familiar" sobre o nível educacional dos indivíduos, enquanto que com um coeficiente de persistência próximo de zero, sugere que a escolaridade entre as gerações não está relacionada.

Enquanto a regressão de mínimos quadrados estima apenas o efeito médio do impacto de uma variável na distribuição condicional de outra variável dependente (por exemplo, educação dos filhos), o uso de regressões quantílicas, introduzido por Koenker e Bassett (1978), é útil para analisar o impacto das variáveis explicativas nos diferentes pontos da distribuição condicional da variável dependente. Tal investigação permite, assim, explorar uma maior quantidade de informa- 
ção estatística presente nos dados, principalmente em situações em que o comportamento médio é pouco representativo.

Alguns autores têm enfatizado que as assimetrias na distribuição de escolaridade podem afetar o grau de mobilidade intergeracional [Paschoal, (2005)]. Por exemplo, como mencionado anteriormente, Checchi (2006) argumenta que restrições de liquidez podem fazer com que famílias pobres sejam inabilitadas a investir na educação de seus filhos. Se isto for verdade, espera-se que a persistência de educação deverá ser diferente, de acordo com o nível educacional dos pais. Dunn (2004), por outro lado, observou que a influência da escolaridade dos pais pode alterar o formato, a média e a dispersão da distribuição de educação dos filhos, afirmando que cada geração tem uma única distribuição que depende da escolaridade de seu pai. Além destes argumentos, como já discutido, a estimação do coeficiente de persistência para vários pontos da distribuição pode informar sobre a importância relativa de fatores não associados à restrição de renda (mais presente entre famílias mais pobres).

Para introduzir tal noção, considere-se $\left(\boldsymbol{y}_{i}, \boldsymbol{x}_{i}\right), i=1, \ldots . ., n$, uma amostra de uma população qualquer, onde $\boldsymbol{x}_{i}$ é um vetor de $K \times 1$ variáveis explicativas. $\mathrm{O} \tau$-ésimo quantil de $\boldsymbol{y}$ é definido como:

$$
F^{-1}=\inf \{y: F(y) \geq \tau\}
$$

onde $\boldsymbol{F}$ é a função de distribuição (não condicionada) de $\boldsymbol{y}$. Como no caso aqui estudado $\boldsymbol{y}$ e $\boldsymbol{x}$ se relacionam linearmente, como na Equação 1, acima. No caso dos quantis condicionais da distribuição de y, definidos a partir dos quantis da distribuição dos erros, verifica-se:

$$
\begin{aligned}
& \operatorname{Pr}\left(y_{i} \leq y / x_{i}\right)=F_{\varepsilon_{\tau}}\left(y-x_{i} \beta_{\tau} / x_{i}\right) \\
& \mathrm{i}=1, \ldots, \mathrm{n}
\end{aligned}
$$

que permite escrever a chamada função quantílica:

$$
Q_{\tau}\left(y_{i} \backslash x_{i}\right)=x_{i} \beta_{\tau}+F_{\varepsilon}^{-1}(\tau)
$$


O estimador $\hat{\beta}_{\tau}$ (5) da função quantílica acima é definido como solução da seguinte função objetivo:

$$
\min _{\beta \in \Re^{k}}\left[\sum_{i \in\left\{i: y_{f i} \geq X \beta\right\}} \tau\left|y_{f i}-X \beta\right|+\sum_{i \in\left\{i: y_{f i}<X \beta\right\}}(1-\tau)\left|y_{f i}-X \beta\right|\right]
$$

Note-se que diferentemente da minimização de quadrados dos desvios, aqui há minimização de valores absolutos. $\mathrm{O}$ modelo especifica a função quantil condicional da variável dependente y, dada a matriz de regressores $X$ como:

$$
\begin{aligned}
& Q_{y_{f}}(\tau \backslash X)=X \beta(\tau) \\
& \tau \in[0,1]
\end{aligned}
$$

Como argumenta Buchinsky (1998), a regressão quantílica apresenta características que a tornam extremamente interessante: pode ser usada para caracterizar toda a distribuição condicional de uma variável resposta dado um conjunto de regressores; tem uma representação na forma de programação linear que facilita a estimação dos parâmetros; a função objetivo da regressão quantílica é uma soma ponderada de desvios absolutos, fornecendo uma medida de locação robusta, de modo que o vetor de coeficientes estimado não é sensível a observações extremas na variável dependente; quando os erros não seguem a distribuição normal, os estimadores de regressão quantílica podem ser mais eficientes que os estimadores de mínimos quadrados; soluções diferentes para quantis distintos podem ser interpretados como diferenças na resposta da variável dependente às mudanças nos regressores em vários pontos da distribuição condicional da variável dependente. 


\section{Mobilidade Intergeracional de Educação: Evidências Empíricas Disponíveis}

Os trabalhos empíricos disponíveis parecem revelar que o grau de persistência intergeracional de educação, em geral, é mais elevado em países da América Latina do que em qualquer outro país que tenha sido estudado, variando de 0,19 na Malásia a 0,70 no Brasil e na Colômbia. A persistência educacional nos Estados Unidos é baixa, variando entre 0,25 e 0,35. Peru e México apresentam um valor intermediário de persistência, em torno de 0,50 . A Tabela 1 , a seguir, apresenta um resumo das estimativas do grau de persistência intergeracional de educação obtidas na literatura através do cálculo das elasticidades educacionais de pais e filhos (quando adultos) em amostras representativas de vários países.

Tabela 1: Estudos de Persistência Intergeracional de Educação na Literatura

\begin{tabular}{lcc}
\hline Autor & Grau de Persistência Educacional & País \\
\hline Borjas (1992) & 0,25 & Estados Unidos \\
Couch e Dunn (1997) & 0,27 & Estados Unidos \\
Mulligan (1997) & 0,32 & Estados Unidos \\
Behrman, Gaviria e Székely (2001) & 0,35 & Estados Unidos \\
Couch e Dunn (1997) & 0,2 & Alemanha \\
Behrman, Gaviria e Székely (2001) & 0,7 & Brasil \\
Behrman, Gaviria e Székely (2001) & 0,7 & Colômbia \\
Behrman, Gaviria e Székely (2001) & 0,5 & México \\
Behrman, Gaviria e Székely (2001) & 0,5 & Perú \\
Lilard e Willis (1994) & 0,19 & Malásia \\
Ferreira e Veloso (2003b) & 0,81 & Brasil \\
\hline
\end{tabular}

Fonte: Ferreira e Veloso (2003b).

Behrman, Gaviria e Székely (2001) estudaram a mobilidade intergeracional de educação nos Estados Unidos e América Latina e encontraram que o grau de mobilidade educacional é consideravelmente menor em países latino-americanos do que em países desenvolvidos. Eles também mostraram que a mobilidade educacional vem aumentando para pessoas mais jovens no Brasil e em outros países da América Latina, tanto para homens como para mulheres. Utilizando-se das PNAD's de 1982 e 1996, os autores encontraram evidências de que o grau de mobilidade intergeracional educacional para homens no Brasil é muito pequeno, com um grau de persistência da ordem de 0,70. Em outras palavras, esses resultados apontam 
que o "background familiar" é muito mais importante no Brasil e na Colômbia para a determinação do nível educacional dos indivíduos (homens) que nos demais países da América Latina e do mundo.

Em outra comparação com países da América Latina, Behrman et al. (1999) utilizam dados de dezesseis países da região para aplicar o conceito de defasagem escolar ${ }^{14}$ que é a diferença entre a escolaridade que o indivíduo deveria ter se tivesse começado a estudar aos seis anos de idade e avançasse uma série por ano e a série que ele efetivamente tem, e relacioná-lo com a escolaridade do pai, mãe, com renda da família e outras variáveis de controle. Os autores observaram que nos países da América Latina, quanto maior a escolaridade dos pais e mães, menor é a defasagem escolar, e encontraram relação negativa entre defasagem escolar e a renda da família, ou seja, quanto maior a renda familiar, menor a defasagem escolar para todos os países analisados.

Dunn (2004) argumenta que o Brasil experimentou uma redução na transmissão de educação e isto se refletiu em queda da transmissão de renda ao longo do tempo. $\mathrm{O}$ autor analisou dois potenciais determinantes da mobilidade intergeracional de renda, os quais, retornos de escolaridade e a mobilidade intergeracional de educação, encontrando que 95\% da variação da mobilidade intergeracional de renda é explicada pela mobilidade intergeracional de educação e pelos retornos de escolaridade. Dunn (2004) concluiu que os retornos da educação explicam as diferenças na mobilidade intergeracional de renda entre as idades e o grau de persistência intergeracional de educação explica as diferenças entre as coortes de nascimento. Além disso, o autor destacou que o aumento na mobilidade intergeracional de renda que o Brasil experimentou nos últimos cinquenta anos do século passado foi resultado do aumento na mobilidade intergeracional de educação.

Os graus de persistência intergeracional de educação estimados por Dunn (2004), sem controles, foram de 0,93 para a PNAD do ano de 1992, 0,87 para a PNAD do ano de 1988 e 0,84 para a PNAD de 1996. Os retornos estimados de um ano a mais de educação na renda foram de 0,16 para os anos de 1982 e 1988 e 0,15 para o ano de 1996. Dunn (2004) e Lam (1999) evidenciam a importância da

${ }^{14} \mathrm{O}$ termo em inglês é o "schooling gap". 
mobilidade intergeracional de educação para explicar as desigualdades na renda.

Utilizando-se de matrizes de transição e estimações de persistência por mínimos quadrados ordinários, Ferreira e Veloso (2003b) apresentam evidências detalhadas de que a mobilidade intergeracional de educação no Brasil é menor que a observada nos países desenvolvidos e em desenvolvimento, com exceção da Colômbia. Em particular, a mobilidade é menor no Nordeste que no Sudeste, e é mais baixa entre negros do que entre brancos. Além disso, a fração de filhos de pais sem escolaridade que permanecem na categoria de educação de seu pai é de cerca de $34 \%$, ao passo que a fração de filhos cujos pais tenham concluído o ensino superior que repetiram o desempenho dos pais é de $60 \%$. Para os autores, grande parte da baixa mobilidade educacional observada para filhos de pais sem escolaridade reflete a dificuldade de ascensão educacional de filhos de pais analfabetos.

Paschoal (2005) investigou em que medida as assimetrias na distribuição de educação são importantes para a determinação do grau de mobilidade intergeracional. Os principais resultados apontaram que, utilizando-se da PNAD de 1996, o grau de persistência educacional no Brasil apresenta um formato de U invertido, ou seja, é maior no centro da distribuição e menor nas caudas; encontrou também que a persistência de educação entre mães e filhos é maior que a persistência educacional entre pais e filhos, tanto para homens quanto para mulheres; e que a mobilidade é menor entre negros e pardos e entre pessoas residentes em áreas rurais.

\section{Bases de Dados}

O conjunto de evidências obtidas e apresentadas neste trabalho deriva da utilização de duas fontes de informações. Primeiro, com base na PNAD de 1996, a amostra é composta de chefes e cônjuges de família com idade entre 23 e 69 anos, que não frequentam escola e que reportaram informações válidas sobre a própria educação e a educação de seus pais. Sob tais condições, 36.478 pessoas relataram sobre a própria educação e sobre a educação do pai, e 37.631 relataram sobre a própria educação e a educação da mãe, distribuídas entre as RMs do Brasil, exceto Distrito Federal. De acordo com Behrman 
et al. (2001), a exclusão dos estudantes é necessária, uma vez que não atingiram o mais alto nível de escolaridade que poderão possuir para que seja comparável com o nível de escolaridade máximo que os pais atingiram.

Uma segunda fonte de informações utilizada corresponde ao banco de dados da Fundação Joaquim Nabuco (Fundaj), construído exclusivamente para a Região Metropolitana do Recife em 2010. Esse banco de dados faz parte de uma linha de pesquisa mais ampla da Fundaj, cujo objetivo é o estudo de mobilidade social na Região Metropolitana do Recife. Os referidos dados foram coletados aos moldes das PNAD's, contendo um banco com informações dos domicílios e outro contendo informações das pessoas, mas numa escala menor, abrangendo apenas a Região Metropolitana do Recife. Entre outras informações (como escolaridade, trabalho e rendimento), esse banco possibilita estudar mobilidade intergeracional de educação, pois contém informações sobre a escolaridade do pai e da mãe quando a pessoa (chefe ou cônjuge de família) tinha 15 anos de idade. ${ }^{15}$

Tal banco é composto de 1.800 domicílios da RM do Recife, abrangendo 5.913 pessoas, das quais 2.935 são classificadas como chefes e cônjuges de família. A condição para que a informação seja utilizada no estudo, é a mesma condição imposta aos dados da PNAD, ou seja, que a pessoa tenha entre 23 e 69 anos de idade, seja não estudante e que tenha relatado informação sobre a própria educação e a educação do pai e da mãe. Sob tais condições, a amostra válida é composta de 1.959 pessoas.

Como a PNAD de 1996 reporta a escolaridade dos pais em categorias descontínuas, a transformação das categorias de escolaridade dos pais ficou assim definida: zero anos de estudo para os que reportaram que nunca frequentaram escola ou não concluíram a primeira série do primeiro grau; dois anos de estudo se completaram da primeira à terceira série do primeiro grau, ou, na nomenclatura atual, possuem o ensino básico incompleto; quatro anos de estudo se completaram o ensino básico; seis anos de estudo se têm ensino fundamental incompleto; oito anos de estudo se completaram o ensino fundamental; dez anos de estudo se não completaram o ensino médio; onze anos de estudo se completaram o ensino médio; treze

15 Note-se que a informação sobre a educação dos pais só é disponível para pessoas classificadas como chefes e cônjuges de família. O mesmo ocorre com os dados das PNAD's. 
anos de estudo se não completaram o ensino superior; e 16 anos de estudo se completaram o ensino superior ou mestrado ou doutorado. Para manter a homogeneidade do estudo, a educação do pai e da mãe com base nos dados da Fundaj foi construída da mesma forma que os dados da PNAD.

Tabela 2: Estatísticas Descritivas Amostrais, Região Metropolitana do Recife1996 e 2010

\begin{tabular}{ccccccc}
\hline & & $\begin{array}{c}\text { Número de } \\
\text { Observações }\end{array}$ & $\begin{array}{c}\text { Média de } \\
\text { Idade }\end{array}$ & $\begin{array}{c}\text { Escolaridade } \\
\text { Média }\end{array}$ & $\begin{array}{c}\text { Escolaridade } \\
\text { do Pai }\end{array}$ & $\begin{array}{c}\text { Escolaridade } \\
\text { da Mãe }\end{array}$ \\
\hline \multirow{2}{*}{1996} & Homens & 2.602 & 38,75 & 6,89 & 3,67 & 2,89 \\
& Mulheres & 3.171 & 39,9 & 6,98 & 3,37 & 2,87 \\
& Total & 5.773 & 39,38 & 6,94 & 3,49 & 2,88 \\
\hline \multirow{2}{*}{2010} & Homens & 765 & 45,91 & 6,96 & 3,77 & 3,13 \\
& Mulheres & 1.194 & 45,27 & 6,82 & 3,15 & 2,79 \\
\hline
\end{tabular}

Fonte: Cálculos dos autores com base em dados da PNAD e Fundaj.

A Tabela 2, acima, apresenta as estatísticas descritivas amostrais para a RM do Recife dos anos de 1996 e 2010, tais como idade, escolaridade média das pessoas e escolaridade dos pais. Com base nessas informações, observa-se que a amostra de 2010 é composta por pessoas mais velhas, com média de idade de 45 anos; as mães de 2010 são mais escolarizadas que as mães de 1996; além desses, houve inversão nos resultados de escolaridade de homens e mulheres, ou seja, na amostra disponível, os homens em 2010 são mais escolarizados que as mulheres; observe-se também que estes resultados apontam a existência de mobilidade absoluta, ou seja, os filhos têm consistentemente superado o nível de escolaridade de seus pais. Note-se que, tanto em 1996 como em 2010, a educação dos filhos é duas vezes superior à educação dos pais. No entanto, este trabalho pretende estudar mobilidade educacional não tanto em termos absolutos, mas em termos relativos. Tal distinção é importante porque a mobilidade absoluta pode ser apenas um reflexo do aumento na média de escolaridade que geralmente acompanha o desenvolvimento econômico. Por outro lado, a mobilidade relativa não é afetada pelo crescimento econômico e está mais relacionada com a distribuição de oportunidades existentes na sociedade, e, portanto, foco de interesse deste estudo. 


\section{Mobilidade Intergeracional de Educação: Estimativas para as Regiões Metropolitanas}

Estimou-se o Modelo (1) sem controles de duas formas. Na primeira, a educação dos filhos era explicada somente pela educação do pai. Esses resultados são apresentados na Tabela 3, a seguir (coluna 1, do Modelo 1). Depois, estimou-se a importância da educação da mãe para a educação dos filhos, resultados mostrados na mesma tabela (coluna 2, Modelo 1). Com base nesses resultados, é possível dizer que a mobilidade educacional nas regiões metropolitanas é maior que a mobilidade educacional no resto do Brasil.

Além disto, a persistência de status educacional entre mães e filhos é maior que a persistência de status educacional entre pais e filhos em todas as regiões. Por fim, a persistência de escolaridade entre pais e filhos, e entre mães e filhos é maior em Curitiba, Belo Horizonte, Recife e Fortaleza, e menor em Belém. Em parte, esses resultados são esperados, uma vez que, como mencionado anteriormente, as regiões metropolitanas são mais dinâmicas em termos de oportunidades educacionais, de emprego e renda, e deste modo favorecendo a mobilidade. Por outro lado, o papel da mulher no cuidado e criação dos filhos é mais importante na sociedade brasileira, e deste modo é de se esperar que a persistência educacional entre mais e filhos seja mais alta que a mesma entre pais e filhos. 
Tabela 3 - Persistência Intergeracional de Educação entre Pais e Filhos e Mães e Filhos - Brasil e Regiões Metropolitanas - 1996 e 2010

\begin{tabular}{|c|c|c|c|c|c|c|c|c|c|c|}
\hline & \multicolumn{2}{|c|}{ Modelo 1} & \multicolumn{2}{|c|}{ Modelo 2} & \multicolumn{2}{|c|}{ Modelo 3} & \multicolumn{2}{|c|}{ Modelo 4} & \multicolumn{2}{|c|}{ Modelo 5} \\
\hline & $\begin{array}{l}\text { Pai e } \\
\text { Filho }\end{array}$ & $\begin{array}{l}\text { Mãe e } \\
\text { Filho }\end{array}$ & $\begin{array}{l}\text { Pai e } \\
\text { Filho }\end{array}$ & $\begin{array}{c}\text { Mãe e } \\
\text { Filho }\end{array}$ & $\begin{array}{l}\text { Pai e } \\
\text { Filho }\end{array}$ & $\begin{array}{c}\text { Mãe e } \\
\text { Filho }\end{array}$ & $\begin{array}{l}\text { Pai e } \\
\text { Filho }\end{array}$ & $\begin{array}{c}\text { Mãe e } \\
\text { Filho }\end{array}$ & $\begin{array}{l}\text { Pai e } \\
\text { Filho }\end{array}$ & $\begin{array}{c}\text { Mãe e } \\
\text { Filho }\end{array}$ \\
\hline $\begin{array}{l}2010 \\
\text { Recife }\end{array}$ & 0,494 & 0,556 & 0,463 & 0,52 & 0,464 & 0,52 & 0,435 & 0,489 & & \\
\hline 1996 & & & & & & & & & & \\
\hline Recife & 0,658 & 0,743 & 0,639 & 0,724 & 0,639 & 0,723 & 0,601 & 0,681 & & \\
\hline Belém & 0,552 & 0,592 & 0,536 & 0,572 & 0,536 & 0,572 & 0,505 & 0,539 & & \\
\hline Fortaleza & 0,65 & 0,766 & 0,634 & 0,747 & 0,635 & 0,747 & 0,583 & 0,688 & & \\
\hline Salvador & 0,62 & 0,722 & 0,611 & 0,71 & 0,611 & 0,71 & 0,547 & 0,642 & & \\
\hline $\begin{array}{l}\text { Belo } \\
\text { Horizonte }\end{array}$ & 0,665 & 0,784 & 0,651 & 0,766 & 0,651 & 0,765 & 0,592 & 0,697 & & \\
\hline $\begin{array}{l}\text { Rio de } \\
\text { Janeiro }\end{array}$ & 0,548 & 0,654 & 0,534 & 0,639 & 0,533 & 0,637 & 0,496 & 0,595 & & \\
\hline São Paulo & 0,651 & 0,738 & 0,626 & 0,707 & 0,625 & 0,706 & 0,594 & 0,671 & & \\
\hline Curitiba & 0,661 & 0,761 & 0,648 & 0,746 & 0,649 & 0,746 & 0,631 & 0,727 & & \\
\hline $\begin{array}{l}\text { Porto } \\
\text { Alegre }\end{array}$ & 0,567 & 0,665 & 0,556 & 0,648 & 0,555 & 0,648 & 0,547 & 0,637 & & \\
\hline Brasil & 0,744 & 0,837 & 0,716 & 0,802 & 0,716 & 0,802 & 0,677 & 0,758 & 0,702 & 0,788 \\
\hline
\end{tabular}

Fonte: Cálculos dos autores com base em dados da PNAD e Fundaj. Todos os coeficientes são significantes com nível de confiança de 95\%.

Modelo 1: sem controles. Modelo 2: controles de idade e idade do filho ao quadrado.

Modelo 3: controles de idade e idade do filho ao quadrado, dummy de sexo (1 é homem).

Modelo 4: controles de idade e idade do filho ao quadrado, dummies de sexo (1 é homem) e de raça ( 1 é branco). Modelo 5: idade, idade do filho ao quadrado, dummies de sexo (1 é homem), raça (l é branco) e de região (Nordeste como referência).

Comparando-se os resultados de 1996 e 2010, percebe-se que a persistência de status educacional entre pais e filhos se reduz, mas ainda é alta. Note-se que os coeficientes de persistência estimados para os Estados Unidos por diversos autores (mencionados anteriormente) para a década de 1990 variou no intervalo entre 0,25 e 0,35, enquanto que no resto da América Latina esse coeficiente oscilava em torno de 0,5. No caso da Região Metropolitana do Recife, o coeficiente estimado para o pai em 1996 é de 0,658, o mesmo coeficiente em 2010 foi de 0,494 (resultado mostrado na Tabela 3, acima, na coluna 1 do Modelo 1). 
A persistência educacional da mãe recifense sobre os filhos em 1996 foi de 0,743. Em 2010, esse número é equivalente a 0,556 (Tabela 3, anterior, coluna 2, Modelo 1).

Conforme sugestão de Mulligan (1997), outras versões do modelo descrito em (1) foram estimadas, adicionando-se variáveis de controle, cujo objetivo é apenas controlar as heterogeneidades nas características pessoais e de localização que poderiam proporcionar resultados de mobilidade intergeracional diferenciados. Nas colunas da Tabela 3, acima, nomeadas por Modelo 2, introduziu-se a idade e idade do filho ao quadrado; o Modelo 3 acrescentou uma dummy para sexo (que assumiu valor 1 para homem, zero caso contrário); o Modelo 4 acrescentou uma dummy de raça (1 para branco, zero caso contrário); o Modelo 5 (estimado somente para o Brasil) foram incluídas dummies de localização regional, em que a região Nordeste foi tomada como referência.

O principal resultado fornecido pelas estimações com variáveis de controle informa que a persistência intergeracional de educação, tanto de pais para filhos quanto de mães para filhos se reduz um pouco mais ao se controlar pela raça. Entretanto, essas reduções são muito pequenas. Note-se que a persistência educacional entre pais e filhos, e mães e filhos é um pouco menor entre brancos principalmente se a pessoa é residente em Salvador, Belo Horizonte e Fortaleza. Tal diferença de persistência entre brancos e não brancos parece ser menor em Porto Alegre. Vale ressaltar que esses resultados são muito importantes. Eles apontam que as oportunidades educacionais às quais as pessoas se defrontam são cada vez menos influenciadas por diferenças de gênero ou raça. Ou seja, no Brasil e RM's, o fator que ainda se mostra de grande relevância para a determinação do nível educacional de um indivíduo é muito mais a posição social que a família ocupa e muito menos as diferenças de gênero ou raça.

Adicionalmente, para obter uma estimativa mais precisa da persistência intergeracional de educação entre pais e filhos em diferentes pontos da distribuição, estimou-se o Modelo (1) pelo método de regressões quantílicas. Como mencionado anteriormente, tal metodologia possibilita analisar o impacto da educação do pai e da mãe nos diferentes pontos da distribuição condicional da educação dos filhos. Esses resultados são apresentados nas Tabelas 4 e 5, em seguida. 
É possível dizer, com base nos resultados, que a persistência intergeracional de educação é menor nos extremos da distribuição de escolaridade dos filhos e maior no centro dela. Ou seja, quanto mais extremo o quantil de escolaridade do filho, menor a influência da escolaridade tanto do pai quanto da mãe. Por outro lado, a influência da escolaridade dos pais se mostra substancial nos quantis centrais da distribuição, indicando que a mobilidade intergeracional de educação é menor nesses quantis. Em outras palavras, a persistência intergeracional de educação entre pais e filhos para as regiões metropolitanas do Brasil apresenta o formato de $\mathrm{U}$ invertido, resultado consistente com Paschoal (2005).

Esses resultados são importantes e parecem confirmar a ideia de Checchi (2006) de existência de restrições culturais e financeiras interferindo nas escolhas educacionais das pessoas. Ou seja, pais mais escolarizados pressionam mais seus filhos para obterem níveis educacionais mais elevados por serem mais conscientes do valor da educação. Esses fatores seriam mais intensos nos níveis intermediários de escolaridade dos pais, fazendo com que os filhos desses pais sejam mais restritos, isto é, recebem mais investimento educacional para obterem pelo menos o nível de escolaridade de seus pais, e deste modo, aumentando a persistência intergeracional de escolaridade para níveis intermediários de escolaridade dos pais.

Tabela 4 - Coeficientes de Persistência de Regressão Quantílica, Variável Independente: Educação do Pai - 1996

\begin{tabular}{ccccccccccc}
\hline Quantis & Recife & Brasil & Belém & Fortaleza & Salvador & $\begin{array}{c}\text { Belo } \\
\text { Horizonte }\end{array}$ & $\begin{array}{c}\text { Rio de } \\
\text { Janeiro }\end{array}$ & São Paulo & Curitiba & $\begin{array}{c}\text { Porto } \\
\text { Alegre }\end{array}$ \\
\hline 5 & 0,36 & 0,18 & 0,45 & 0,13 & 0,33 & 0,5 & 0,36 & 0,33 & 0,38 & 0,45 \\
15 & 0,69 & 0,69 & 0,64 & 0,69 & 0,69 & 0,64 & 0,57 & 0,63 & 0,57 & 0,5 \\
25 & 0,67 & 0,86 & 0,56 & 0,64 & 0,63 & 0,56 & 0,5 & 0,5 & 0,5 & 0,5 \\
35 & 0,71 & 0,75 & 0,62 & 0,75 & 0,67 & 0,5 & 0,5 & 0,5 & 0,5 & 0,5 \\
45 & 0,71 & 0,75 & 0,64 & 0,73 & 0,64 & 0,6 & 0,54 & 0,5 & 0,63 & 0,56 \\
55 & 0,64 & 0,67 & 0,43 & 0,64 & 0,55 & 0,64 & 0,55 & 0,73 & 0,67 & 0,55 \\
65 & 0,75 & 0,88 & 0,33 & 0,75 & 0,5 & 0,75 & 0,36 & 0,71 & 0,75 & 0,5 \\
75 & 0,5 & 0,75 & 0,17 & 0,5 & 0,38 & 0,5 & 0,38 & 0,5 & 0,5 & 0,5 \\
85 & 0,33 & 0,75 & 0,08 & 0,25 & 0,08 & 0,75 & 0,25 & 0,75 & 0,5 & 0,25 \\
95 & 0,19 & 0,27 & 0,19 & 0,18 & 0,19 & 0,22 & 0,19 & 0,25 & 0,33 & 0,25 \\
\hline
\end{tabular}

Fonte: Cálculos dos autores com base em dados da PNAD 
A comparação regional permite indicar as seguintes relações. Entre os filhos menos escolarizados, a influência da educação do pai é muito mais alta em Belo Horizonte, Belém e Porto Alegre, e muito mais baixa em Fortaleza; para os filhos de escolaridade mais elevada, a influência da escolaridade do pai é maior em Curitiba e menor em Fortaleza. Já a mobilidade intergeracional de educação é muito menor para os filhos de escolaridade média em Fortaleza e Recife. Em outras palavras, os resultados apontam que, comparativamente aos resultados obtidos para as outras RM's, em Fortaleza a mobilidade educacional é muito maior para filhos mais e menos escolarizados, e muito menor para os filhos de escolaridade média. Os coeficientes de persistência de educação do pai são apresentados na Tabela 4, anterior.

Comparando-se regionalmente os resultados das mães, observa-se que a persistência educacional entre mães e filhos menos escolarizados é menor no Brasil e maior nas regiões metropolitanas, ou seja, nas RM's o efeito da baixa escolaridade das mães sobre os filhos é maior que no restante do país. A persistência intergeracional de educação entre mães e filhos é muito mais baixa em Fortaleza que nas demais RM's nos extremos da distribuição, sendo inexistente para filhos pouquíssimos escolarizados, ou seja, para esses filhos a mobilidade é máxima. Entre os filhos com nível de escolaridade mediano, apenas é possível dizer que, em geral, a persistência em todas as RM's é muito alta, e parece ser menor em Porto Alegre e Belém. Os coeficientes de persistência da mãe são apresentados na Tabela 5 , a seguir.

Tabela 5 - Coeficientes de Persistência de Regressão Quantílica, Variável Independente: Educação da Mãe - 1996

\begin{tabular}{ccccccccccc}
\hline Quantis & Recife & Brasil & Belém & Fortaleza & Salvador & $\begin{array}{c}\text { Belo } \\
\text { Horizonte }\end{array}$ & $\begin{array}{c}\text { Rio de } \\
\text { Janeiro }\end{array}$ & São Paulo & Curitiba & $\begin{array}{c}\text { Porto } \\
\text { Alegre }\end{array}$ \\
\hline 5 & 0,4 & 0,3 & 0,4 & 0 & 0,5 & 0,5 & 0,5 & 0,38 & 0,5 & 0,55 \\
15 & 0,69 & 0,75 & 0,64 & 0,69 & 0,83 & 0,75 & 0,67 & 0,69 & 0,69 & 0,56 \\
25 & 0,75 & 0,16 & 0,64 & 1 & 0,75 & 0,63 & 0,5 & 0,5 & 0,64 & 0,5 \\
35 & 0,73 & 0,82 & 0,73 & 0,82 & 0,73 & 0,67 & 0,67 & 0,56 & 0,57 & 0,57 \\
45 & 0,64 & 0,73 & 0,6 & 0,73 & 0,7 & 0,64 & 0,64 & 0,64 & 0,64 & 0,64 \\
55 & 0,75 & 0,75 & 0,43 & 0,75 & 0,75 & 0,75 & 0,73 & 0,88 & 0,8 & 0,7 \\
65 & 0,75 & 1 & 0,33 & 0,75 & 0,5 & 0,75 & 0,5 & 0,67 & 0,75 & 0,63 \\
75 & 0,6 & 0,83 & 0,17 & 0,67 & 0,5 & 0,67 & 0,5 & 0,67 & 0,64 & 0,63 \\
85 & 0,5 & 0,75 & 0 & 0,5 & 0,17 & 0,75 & 0,25 & 0,75 & 0,75 & 0,43 \\
95 & 0,25 & 0,33 & 0,25 & 0,13 & 0,25 & 0,33 & 0,27 & 0,27 & 0,25 & 0,27 \\
\hline
\end{tabular}

Fonte: Cálculos dos autores com base em dados da PNAD 
Ao se compararem os resultados de persistência para a Região Metropolitana de Recife ao longo do tempo percebe-se que os coeficientes de persistência continuam apresentando o formato de $\mathrm{U}$ invertido, mas com uma pequena diferença: em 2010 a influência da escolaridade dos pais sobre a escolaridade dos filhos cresce nos extremos da distribuição de escolaridade dos filhos e diminui para os filhos de escolaridade intermediária, sugerindo que a mobilidade intergeracional de educação no período mais recente é menor para filhos com pouca e muita escolaridade e aumenta para filhos de escolaridade intermediária. Os resultados de persistência intergeracional de educação entre pais e filhos para a RM de Recife em 1996 e 2010 podem ser visualizados na Figura 1, a seguir.

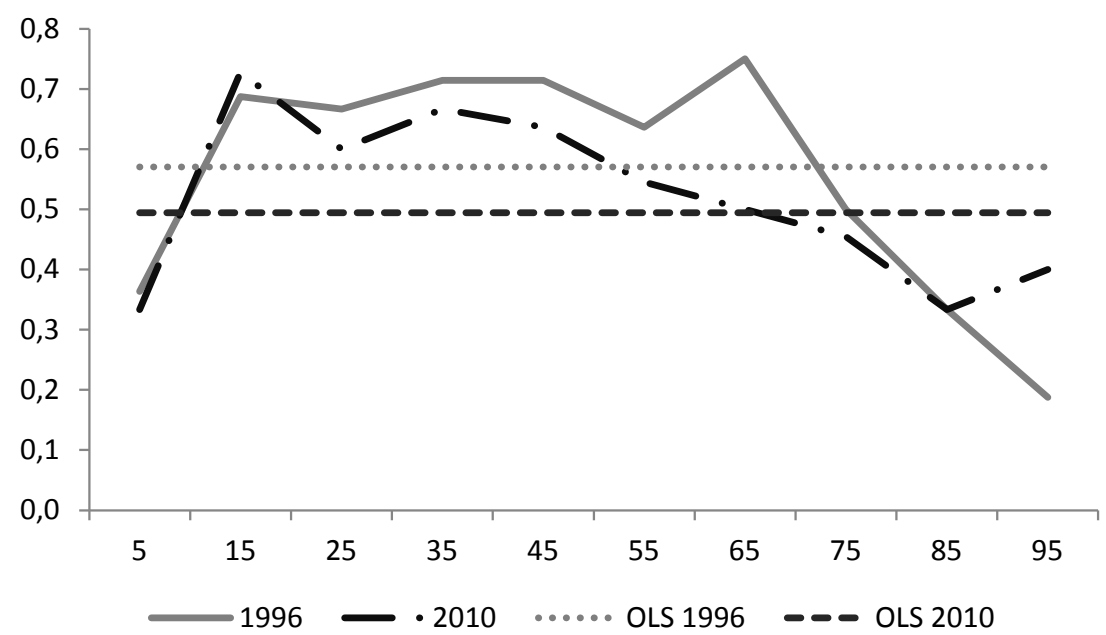

Figura 1 - Persistência Intergeracional de Educação, Variável Independente: Educação do Pai - Região Metropolitana de Recife

Fonte: Cálculos dos autores com base em dados da PNAD e Fundaj.

A mesma tendência da evolução da persistência entre pais e filhos ocorre entre mães e filhos ao longo do tempo, ou seja, no período mais recente a mobilidade parece aumentar para filhos de escolaridade intermediária e diminuir para filhos muito e pouco escolarizados. A persistência entre mães e filhos continua com formato de $\mathrm{U}$ invertido, e permanece mais alta em 2010 que a persistência do pai. 
Os resultados sobre a mobilidade intergeracional de educação entre mães e filhos recifenses ao longo do tempo são apresentados na Figura 2, a seguir.

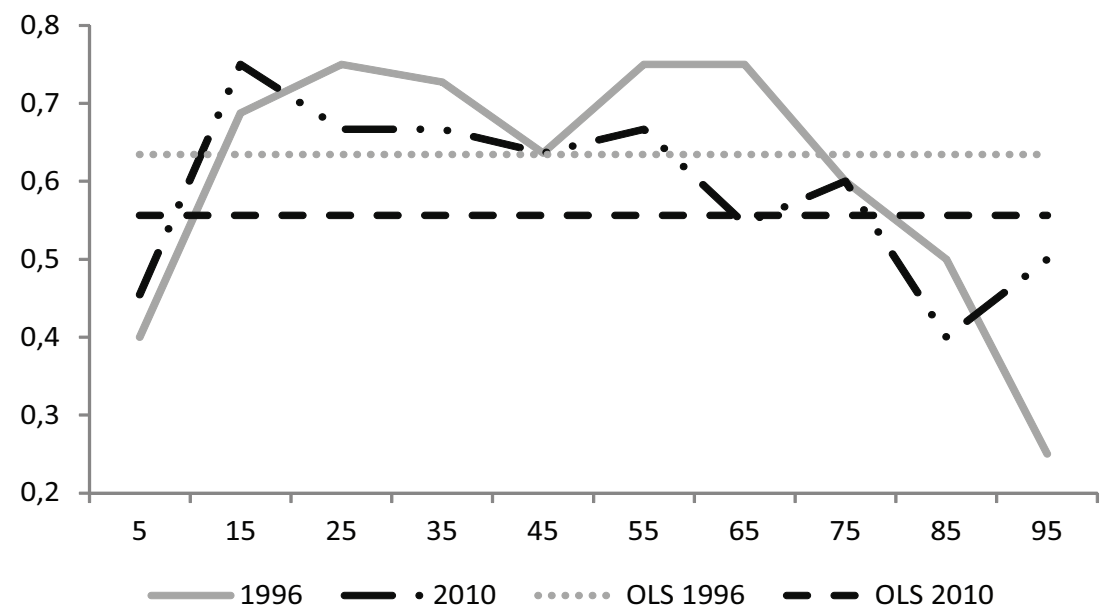

Figura 2 - Persistência Intergeracional de Educação, Variável Independente: Educação da Mãe - Região Metropolitana de Recife

Fonte: Cálculos dos autores com base em dados da PNAD e Fundaj.

\section{Considerações Finais}

O principal objetivo do trabalho foi verificar se as transformações sociais ocorridas no Brasil nas últimas décadas, sobretudo o bom desempenho da economia brasileira em período recente, impactaram positivamente nas oportunidades, refletindo-se no aumento nos índices de mobilidade educacional. Neste sentido, este trabalho fornece resultados sobre a mobilidade intergeracional de educação nas regiões metropolitanas do Brasil em 1996 e principalmente com resultados para um período mais recente para a RM do Recife, 2010, com base em dados inéditos da Fundaj.

Os principais resultados apresentados neste trabalho confirmam o que a literatura que trata da mobilidade intergeracional de educação destaca como importante: a persistência de educação entre pais e filhos no Brasil é muito alta e heterogênea, mesmo em 2010 no caso 
do Recife. Além disso, verificou-se que a persistência educacional nas regiões metropolitanas do Brasil é menor que no resto do país, resultado consistente com Bichernall (2001), em que o efeito das características socioeconômicas dos pais sobre os filhos deve ser menor em regiões mais dinâmicas. Ademais, o efeito da escolaridade da mãe sobre os filhos parece mais importante que o efeito da escolaridade do pai, resultado que pode estar indicando que, uma vez que a mulher tem um papel fundamental na criação e cuidados com os filhos, políticas públicas que visem o aumento das oportunidades e redução das desigualdades podem ser mais eficientes se forem focadas nas mulheres, ou mães.

Comparando-se os resultados de 1996 e 2010, percebe-se que a persistência entre pais e filhos se reduz, mas ainda é alta na RM do Recife. Note-se que os coeficientes de persistência estimados para os Estados Unidos por diversos autores para a década de 1990 variou no intervalo entre 0,25 e 0,35, enquanto que no resto da América Latina esse coeficiente oscilava em torno de 0,5. No caso da Região Metropolitana do Recife, o coeficiente estimado para o pai em 1996 é de 0,658, já o mesmo coeficiente estimado para 2010 foi de 0,494 . No mesmo sentido, a elasticidade estimada da persistência da escolaridade da mãe recifense sobre a escolaridade dos filhos em 1996 foi de 0,743 , valor mais elevado que aquele obtido em 2010, cerca de 0,556 .

Os resultados encontrados com base em regressões quantílicas confirmam a hipótese de Checchi (2006), segundo a qual restrições culturais e financeiras fazem com que a mobilidade intergeracional seja diferente de acordo com o nível educacional dos pais. Filhos de pais muito escolarizados são mais restritos, ou seja, recebem mais investimento educacional e têm também nível de instrução mais elevado, fazendo com que a persistência educacional seja maior para essas pessoas. Encontraram-se também evidências que confirmam a hipótese de Ferreira e Veloso (2003b), mesmo para o caso da RM de Recife em 2010, em que existe uma dificuldade de ascensão educacional de filhos de pais analfabetos.

Destaque-se, por fim, que as evidências encontradas neste trabalho indicam que as oportunidades educacionais às quais as pessoas se defrontam são cada vez menos influenciadas por questões de gênero ou raça, e o fator que ainda se mostra de grande relevância 
para a determinação do nível educacional de um indivíduo é muito mais relacionado com a posição social que a família ocupa. Esses resultados evidenciam que políticas sociais que almejem o aumento das oportunidades de modo a quebrar com o ciclo intergeracional de pobreza poderiam ser mais eficientes se forem concentradas em famílias pobres.

\section{Referências}

A Dictionary of Sociology. John Scott and Gordon Marshall. Oxford University Press 2005.

ALDRIDGE, S. The Facts about social mobility: a survey of recent evidence on social mobility and its causes. New Economy, 10:4, pp.189-193, 2003.

ANDRADE, E. et al. Do Borrowing Constraints Decrease Intergenerational Mobility in Brazil? A Test Using Quantile Regression. IBMEC Working Paper, 2003. Disponível em $<$ http:www.ibmecsp. edu.br/pesquisa/download.php?recid=2544>

ASHENFELTER, O. C.; KRUEGER, A. B. Estimates of the Economic Return to Schooling from a New Sample of Twins. NBER Working Paper Series, Vol. w4143, 1992.

BARROS, R.; CARVALHO, M.; FRANCO, S.; MENDONÇA, R. Uma análise das principais causas da queda recente na desigualdade de renda brasileira. Econômica, Rio de Janeiro, v.8, n.1, p.117147, junho 2006.

BARROS, R. P. de; HENRIQUES, R.; MENDONÇA, R. Desigualdade e pobreza no Brasil: retrato de uma estabilidade inaceitável. Revista Brasileira de Ciências Sociais, n. 42, fev. 2000.

BARROS, R. P.; MENDONÇA, R.; SANTOS, D. D. dos; QUINTAES, G. Determinantes do desempenho educacional no Brasil. Pesquisa e Planejamento Econômico, Rio de Janeiro, v. 31, n. 1, p. 1-42, 2001

BECKER, G. Human Capital: A Theorical and Empirical Analysis, with Special Reference to Education. Chicago: University of Chicago Press [ $1^{\text {st }}$ ed., 1964], 1993.

BECKER, G.; TOMES, N. An equilibrium theory of distribution of income and intergenerational mobility. Journal of Political Economy, 87:6, December, pp.1153-1189, 1979.

. Human capital and the rise and fall of families. Journal of Labor Economics, 4:3, Part2, pp.S1-39, 1986.

BEHRMAN, J. Social mobility: concepts and measurement. In: BIRDSALL,N.; GRAHAM, C. (eds.). New markers, new opportunities? Economic and social mobility in a changing world. Washington: Brookings Institution Press and the Carnegie Endowment for International Peace, 2000.

BEHRMAN, J. et al. Intergenerational mobility in Latin America: Deeper markets and better schools make a difference. Revision of paper presented at Brookings Institution Center on Social and Economics Dynamics/Inter-American Development Bank Workshop on Social Mobility, 1999.

BEHRMAN, J.; GAVIRIA, A.; SZÉKELY, M. Intergenerational mobility in Latin America. Econômica, v.2, n.1, p.1-44, 2001.

BIRCHENALL, J. A. Income distribution, human capital and growth economic: some new evidence. The Economic Journal. v.112, 2001.

BLACK, S.E.; DEVEREUX, P.J. Recent Developments in Intergenerational Mobility. Working Paper. IZA DP No. 4866, 2010. 
BORJAS, G. Ethnic capital and intergenerational mobility. Quarterly Journal of Economics, v.107, n.1, p.123-50, 1992.

BOURDIEU, P. Le capital social: notes provisoires. Actes de la Recherche en Sciences Sociales, n. 31, pp.2-3, 1980.

BOWLES, S.; GINTIS, H. The Inheritance of Inequality. Journal of Economic Perspectives 16 (3), 3-30, 2002.

BUCHINSKY, M. Recent advances in quantile regression models: a practical guideline for empirical research. Journal of Human Resources, 33, p.88-126, 1998.

CHECCHI, D. The Economics of Education. Cambridge: Cambridge University Press, 2006.

CHECCHI, D. DADANONI, V. Mobility comparisons : does using different measures matter? [S.I.], jan. 2002. Available at http://ideas.repec.org/p/mil/wpdepa/2002-15.html.

COUCH, K.; DUNN, T. Intergenerational correlations in labor market status: a comparison of the United States and Germany. Journal of Human Resources, v.32, n. 1, p. 210-232, 1997.

COUCH, K.; LILLARD, D. Nonlinear patterns of intergenerational mobility in Germany and the United States. In M. Corak (Ed.). Generational Income Mobility in North America and Europe. Cambridge: Cambridge University Press, 190-206, 2004.

DEININGER, K.; SQUIRE, L. A New Data Set Measuring Income Inequality. World Bank Economic Review, 10, pp.565-591, 1996.

DUNN, C. The intergenerational transmission of earnings: evidence from Brazil. University of Michigan (Technical Report), 2004.

FERREIRA, S.; VELOSO, F. Mobilidade intergeracional de educação no Brasil. In: XXXI Encontro da Associação Nacional de Centros de Pós-Graduação em Economia, 2003, Porto Seguro - Bahia. Anais do XXXI Encontro de Associação Nacional de Centros de Pós-Graduação em Economia, 2003b.

FRIEDMAN, M. Capitalism and Freedom. [S.I.]: Princeton, 1962.

GALOR, O.; ZEIRA, J. Income distribution and macroeconomics. Review of Economics Studies, v.60, 1993.

GRAWE, N. Intergenerational mobility for whom? The experience of high-and low-earning sons in international perspective. In M. Corak (Ed.). Generational Income Mobility in North America and Europe. Cambridge: Cambridge University Press, 59-89, 2004.

HEATH, A.; PAYNE, C. Twentieth Century trends in social mobility in Britain, Centre for Research into Elections and Social Trends, Working Paper 70, Junho 1999.

HOFFMANN, R. Transferência de renda e a redução da desigualdade no Brasil e cinco regiões entre 1997 e 2004. Econômica, v. 8, n.1. pp. 55-81, Junho 2006. Disponível em: <http://www.uff.br/ cpgeconomia/economica.htm>.

HOLMLUND, H.; LINDAHL, M.; PLUG, E. The Causal Effect of Parents' Schooling on Children's Schooling: A Comparison of Estimation Methods. Journal of Economic Literature 49:3, 615-651, 2011.

KOENKER, R.; BASSETT JR., G. Quantile Regression. Econometrica, Vol. 46, Nº1, pp.33-50, 1978.

LAM, D. Generating extreme inequality: schooling, earnings and intergenerational transmission of human capital in South Africa and Brazil. Population Studies Center Research Report, n.99, 1999.

LAM, D.; SCHOENI, R. F. Effects of family background on earnings and returns to schoolings: evidence from Brazil. Journal of Political Economy, v. 101, n. 4, p.710-741, 1993.

LILLARD, L., WILLIS, R. Intergenerational educational mobility: effects of family and state in Malaysia. Journal of Human Resources, v. 29, n.4, p.1126-1166, 1994.

MULLIGAN, C. Parental priorities and economic inequality. Chicago: University of Chicago Press, 1997. 
PASCHOAL, I. P. Mobilidade Intergeracional de Educação no Brasil. Monografia (Trabalho de Conclusão de Curso de Economia) - Faculdade de Economia, Administração e Contabilidade de Ribeirão Preto, Universidade de São Paulo, Ribeirão Preto, 2005.

PASTORE, J. Desigualdade e mobilidade Social no Brasil. São Paulo: Editora da Universidade de São Paulo, 1979.

. Desigualdade e mobilidade social: dez anos depois. In: BACHA, E.; KLEIN, H. (eds.). A transição incompleta: Brasil desde 1945. Rio de Janeiro: Paz e Terra, 1986.

PASTORE, J.; SILVA, N. V. Mobilidade Social no Brasil. Makron Books, 1999.

PASTORE, J.; ZYLBERSTAJN, H. Social mobility: the role of education in determining status. In: BIRDSALL, N.; SABOT, R. (eds.). Opportunity foregone: education in Brazil. Washington: Inter-American Development Bank, p. 289-318, 1996.

PERO, V.; SZERMAN, D. Mobilidade Intergeracional de Renda no Brasil. Pesquisa e Planejamento Econômico, IPEA, 2006.

PNUD. Programa das Nações Unidas para o Desenvolvimento: Relatório do Desenvolvimento Humano 2006. 2006. Disponível em: <http://www.pnud.org.br/arquivos/rdh/rdh2006/rdh2006_brasil.pdf > .

PRAIS, S. Social mobility and redistributive politics. Journal of the Royal Statistical Society Series A, v.118, n.1, p.56-66, 1955.

PRONZATO, C. An examination of paternal and maternal intergenerational transmission of schooling. Journal of Population Economics 25, 591-608, 2012.

ROCHA, S. Pobreza no Brasil, afinal de que se trata? Rio de Janeiro: FGV, 2003a.

SCALON, M. C. Mobilidade Social no Brasil: Padrões e Tendências. Rio de Janeiro: Revan: IUPERJ-UCAM, 1999.

SHAVIT, Y.; BLOSSFELD, H. (Ed.). Persistent Inequality: Changing Educational Stratification in Thirteen Countries. Boulder, CO: Westview Press, 1993.

SIMON, C. P.; BLUME, L. Matemática para Economistas. [S.1.:s.n.], 2004.

SOARES, S. S. D. Análise de bem-estar e decomposição por fatores da queda na desigualdade entre 1995 e 2004. Econômica, Rio de Janeiro, v.8, n.1, p.83-115, junho 2006. 